\title{
The Influence of Geometry on the Thermal Performance of Microchannels in Laminar Flow with Viscous Dissipation
}

\author{
Marco LORENZINI ${ }^{1, *}$, Nicola SUZZI $^{1}$ \\ * Corresponding author: Tel.: +39 0543374455; Fax: +39 0543374444 2; Email: marco.lorenzini@unibo.it \\ 1 Department of Industrial Engineering - DIN Alma Mater Studiorum, Bologna, Forlì Campus, I
}

\begin{abstract}
Micro heat exchangers (MHXs) may achieve very high heat transfer coefficients thanks to their small dimensions and high Area-to-Volume ratio even in laminar flow. The main drawback of these devices is the high frictional losses - especially for liquid flows - that make viscous dissipation no longer negligible. In order to enhance heat transfer, modification of the channels' cross-section is a viable strategy. In the present work the fully developed steady laminar flow of a Newtonian liquid through a microchannel subject to $\mathrm{H} 1$ boundary conditions in the presence of viscous dissipation is investigated. Entropy generation numbers and FG1a performance evaluation criterion are employed to assess the influence of smoothing the corners of an initially rectangular cross-section, with an aspect ratio ranging from 1 to 0.03 under four different types of geometrical constraints. The governing equations and the results are expressed in non-dimensional form, the intensity of viscous dissipation being exemplified by the Brinkman number, which is demonstrated to increase its maximum allowable value when corners are smoothed. The results are reported as a function of the non-dimensional radius of curvature $R_{c}$ and aspect ratio and show that smoothing the corners almost invariably brings about a benefit for a fixed heated perimeter.
\end{abstract}

Keywords: Micro heat exchangers, viscous dissipation, entropy minimization, PEC.

\section{Introduction}

Microchannels are the building block of many micro flow devices (MFDs), among which micro heat exchangers (MHXs) represent a significant group. The small dimensions allow very high heat transfer coefficients to be reached even in laminar flow, and this, together with a high area-tovolume ratio make MHXs attractive in many applications (Dessiatoun et al. 2013, Han et al. 2011, Kew and Reay 2011). The main drawback of these devices is represented by their high frictional losses - especially for liquids - that affect the flow even under laminar conditions; this not only increases the pressure drop that the flowing fluid experiences, but also lessens the heat duty because of viscous heating. The latter was responsible for many of the apparently novel results of early experiments, as pointed out by Morini (2005). Like for heat exchangers with channels of the more conventional sizes, there is a constant drive towards enhanced performance for MHXs too, usually in terms of higher heat duty, which is accompanied by increased pressure losses. Finding a satisfactory balance between the two conflicting objectives under given constraints is a widely studied engineering problem (Jaluria 2007). To enhance heat transfer, several strategies may be adopted at the macroscale, (an introductory, wide overview is given by Webb 1981) but for MHXs actions are limited by technological barriers such as the difficulty of inserting turbulence promoter within the channels, so that modification of the channel's morphological characteristics, is a much more viable solution. Owing to the manufacturing processes employed, one such modification is that of the channels' crosssection, see Lorenzini and Morini, 2011. The extent to which a given change from the original conditions (which act as reference values) brings about an enhancement in the quantity which one aims at maximizing (objective function) is usually rated through performance evaluation criteria (PECs). PECs are obtained from an analysis in terms of the first law of thermodynamics, as suggested by 
Bergles and Webb $(1981,1983)$. Thanks to the decisive and extensive contributions of Bejan (e.g. 1980, 1984), also criteria based on the second law of thermodynamics have been used in more recent years. Currently, both approaches are considered when optimizing the channels of heat exchangers, see e.g. the large body of works by Zimparov, who combined and extended the two previous methods (2001) and co-authors. An extensive list of their publications on the subject can be found in Zimparov et al. (2014).

In spite of the many works to assess the performance of other cross-sectional shapes compared to that of cylindrical ducts, little attention has been devoted to the influence of smoothing of the corners. Ray and Misra (2006) and Chakraborty and Ray (2013) have focused their attention on square ducts to compute the Poiseuille number, $f R e$, and the Nusselt number, $N u$, for different radii of curvature and $\mathrm{H} 1$ and $\mathrm{H} 2$ boundary conditions, then assessing their performance in terms of objective functions and entropy generation numbers, but they never took viscous heating into account. Lorenzini and Morini (2011) and Lorenzini (2013) extended the analysis of the heat transfer and friction losses, when corners are progressively rounded in rectangular ducts, calculating $f R e$ and $N u$ without and with viscous heating accounted for. This latter effect is promoted in the case of microchannels owing to the reduction in the typical characteristic dimensions involved and is more serious for liquids, although the results presented here are also valid for the case of an incompressible gas flow with thermophysical properties unaffected by the temperature variations experienced.

The present work extends the scope of the latter investigations by employing first and second-law related criteria. The steady, fully developed laminar flow of a Newtonian fluid through a microchannel subject to $\mathrm{H} 1$ (uniform heat flux and uniform temperature along the heated perimeter of the cross-section) in the presence of viscous dissipation is studied. Entropy generation numbers and PECs are employed to assess the influence of smoothing the corners of an initially rectangular cross- section, with an aspect ratio (shorter side, $2 a$ over longer side, $2 b), \beta$, ranging from 1 (square channel) to 0.03 (similar to two parallel plates) under four different types of geometrical constraints. Governing equations and results are given in non-dimensional form, in order to lend a general character to the treatment. The intensity of viscous heating is exemplified by the Brinkman number, which is demonstrated to increase its maximum allowable value when corners of the crosssection are smoothed. Four geometrical constraints are applied because it can be demonstrated that the resulting objective functions and entropy generation numbers are not only dependent on $\beta$ and on $r_{c}$, but also of the ratio a/aref (the subscript indicating the reference value), unless some extra condition is given so that the ratio can be expressed in terms of $\beta$ and $R_{c}=r_{c} / a$ too. The four resulting geometrical constraints are: constant characteristic length, i.e. $a / a_{r e f}=1$, constant hydraulic diameter, i.e. $D_{h}{ }^{*}=1$, constant heated perimeter, i.e. $P_{h}{ }^{*}=1$, and constant crosssectional area, i.e. $A_{c}{ }^{*}=1$. It is to be mentioned here that all symbols bearing a superscript ' $*$ ' indicate a normalized quantity obtained dividing its dimensional counterpart by its reference value.

Only one performance evaluation criterion is investigated in the present study due to space limitations, namely the one known as FG1a, which is described at the beginning of section 3.1. The entropy generation number is also studied and the contributions of its two components (entropy generation due to frictional losses and to heat transfer) are discussed. To combine the results of the two approaches, the method suggested by Zimparov (2001) is used.

The results are reported versus the nondimensional radius of curvature in normalized form taking the values for sharp corners $\left(R_{c}=0\right)$ under the same geometrical constraints as reference.

\section{Statement of the problem}

\subsection{Geometry and problem conditions}

The reference geometry which was studied is a 
channel with axially uniform rectangular cross-section, whose corners are progressively rounded, see Fig.1.

The section is identified by the aspect ratio $\beta=2 a / 2 b$, which takes the values $\beta=1,0.50$, $0.25,0.10,0.03$, ranging from a square duct to a thin slot, similar to two parallel plates.

For a given aspect ratio, calculations are made starting from the base geometry and progressively smoothing the corners, increasing the value of the radius of curvature $r_{c}$, which is made non-dimensional by dividing it by the half shorter side, i.e. $R_{c}=r_{c} / a$.

To avoid undercuts, the maximum value of $r_{c}$ is set at $a$, so that $0 \leq R_{c} \leq 1$ for all cases.

It is assumed that an incompressible, Newtonian fluid flows through the duct in steady, fully-developed, laminar regime, subject to no-slip at the walls and $\mathrm{H} 1$ thermal boundary conditions (uniform heat flux and uniform temperature along heated perimeter $P_{h}$ of the cross-section) on all four sides.

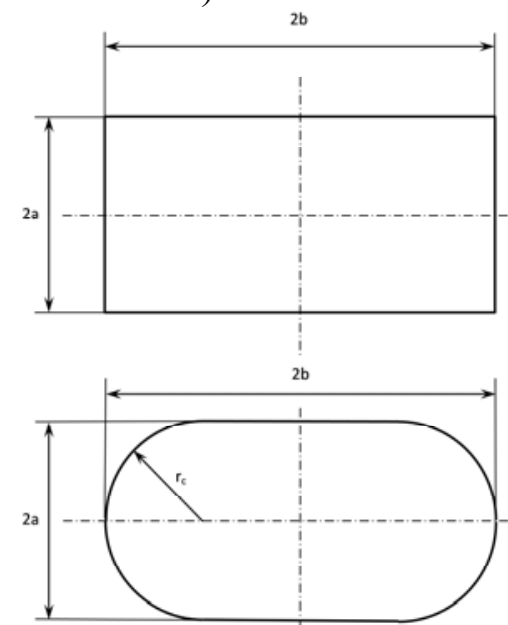

Figure 1 - Base geometry (above) and modified geometry (below), $\beta=0.5$

Viscous dissipation is quantified by the Brinkman number, $B r$, but thermophysical properties are assumed to be constant. For these conditions, the velocity and temperature fields are known and the Poiseuille and Nusselt numbers are those computed by Lorenzini (2013).

\subsection{Entropy Balance Equations}

For an elementary length of a duct, the entropy generation rate $\dot{S}_{g e n}$ under steady state conditions is the sum of one contribution from heat transfer and one from the pressure drop

$$
d \dot{S}_{g e n}=d \dot{S}_{g e n_{\Delta T}}+d \dot{S}_{g e n_{\Delta p}}
$$

The first quantity on the RHS is (Suzzi 2014)

$$
d \dot{S}_{g n_{\Delta T}}=\dot{m} c d T_{m}\left[\frac{1}{T_{m}}-\frac{1}{(1+2 B r f \operatorname{Re})\left(T_{m}+\Delta T\right)}\right]
$$

with $\dot{m}$ mass flow rate, $c$ the thermal capacity of the fluid, $T_{m}$ the bulk temperature and $\Delta T$ the temperature difference between wall and bulk temperature. The second quantity is

$$
d \dot{S}_{g e n_{\Delta p}}=-\frac{\dot{m}}{\rho T_{m}} d p
$$

Where $\rho$ is the fluid density and $p$ is the local pressure. Under the assumptions

$$
\begin{aligned}
& \frac{\Delta T}{T_{m}}<<1 \\
& \frac{T_{e}-T_{i}}{T_{i}}<<1
\end{aligned}
$$

with $T_{e}$ the fluid temperature at the outlet and $T_{i}$ the fluid temperature at the inlet. Equation (1) is integrated over the duct's length to yield

$$
\dot{S}_{g e n_{\Delta T}}=\frac{\dot{Q}_{w} \Delta T}{T_{i}^{2}\left(T_{e} / T_{i}\right)}\left[1+2 B r \frac{A_{c}}{D_{h}^{2}} f \operatorname{Re}\left(1+\frac{T_{e}}{\Delta T}\right)\right]
$$

where $\dot{Q}_{w}$ is the total heat duty (heat transfer rate to the fluid), $A_{c}$ the cross-sectional area and $D_{h}$ the hydraulic diameter. Equation (5) holds for all values of $r_{c}$, and may be normalized by dividing it by its value in a reference configuration (in this case the crosssection for sharp corners). The entropy generation number (Bejan 1984) due to thermal phenomena, is thus

$N_{T}=\frac{\dot{S}_{\text {gen }_{\Delta T}}}{\left(\dot{S}_{\text {gen }_{\Delta T}}\right)_{\text {ref }}}$

and its expression is

$$
N_{T}=\frac{\dot{Q}^{*} \Delta T^{*}}{T_{e}^{*}} \frac{1+2 B r \frac{A_{c}}{D_{h}{ }^{2}} f \operatorname{Re}\left[1+\frac{T_{e}^{*}}{\Delta T^{*}}\left(\frac{T_{e}}{\Delta T}\right)_{r e f}\right]}{1+2 B r_{r e f}\left(\frac{A_{c}}{D_{h}{ }^{2}}\right)_{r e f}(f \operatorname{Re})_{r e f}\left[1+\left(\frac{T_{e}}{\Delta T}\right)_{r e f}\right]}
$$

The non-dimensional exit temperature is obtained from direct integration of the first law energy balance for a channel length $d x$ over the whole length of the channel

$$
T_{e}^{*}=\frac{T_{i}}{\left(T_{e}\right)_{r e f}}+\left(1-\frac{T_{i}}{\left(T_{e}\right)_{r e f}}\right) \frac{\dot{Q}^{*}}{\dot{m}^{*}} \frac{1+2 B r \frac{A_{c}}{D_{h}^{2}} f \mathrm{Re}}{1+2 B r_{r e f}\left(\frac{A_{c}}{D_{h}^{2}}\right)_{r e f}(f \mathrm{Re})_{r e f}}
$$

Concerning entropy generation due to pressure 
losses, its expression is unchanged from the case when no viscous dissipation is present, owing to the assumption of thermal properties independent of pressure and temperature for the ranges encountered in the analysis, so that

$\mathrm{N}_{\mathrm{P}}=\mathrm{P}^{*}=\frac{\mathrm{P}}{\mathrm{P}_{\text {ref }}}=\frac{\dot{\mathrm{m}}^{* 2} \mathrm{~L}^{*}(\mathrm{fRe})^{*}}{\mathrm{~A}_{\mathrm{c}}^{*} \mathrm{D}_{\mathrm{h}}^{* 2}}$

where $P$ is the pumping power needed to maintain the desired flow rate. Introducing the augmentation irreversibility ratio, $\phi$ (Bejan 1984)

$\phi=\frac{\dot{S}_{g e n_{\Delta p}}}{\dot{S}_{g e n_{\Delta T}}}$

The entropy generation number $N_{s}$ becomes

$N_{S}=\frac{\dot{S}_{g e n}}{\left(\dot{S}_{g e n}\right)_{r e f}}=\frac{N_{T}+\phi_{r e f} N_{P}}{1+\phi_{r e f}}$

For this work the value of $T_{i} /\left(T_{e}\right)_{r e f}$ is set to 0.95 , the reference irreversibility ratio $\phi_{\text {ref }}$ is assumed to be $10^{-1}$ and the ratio $T_{e} / \Delta T$ is taken equal to 20. The reference Brinkman number, $\mathrm{Br} r e f$, is that corresponding to sharp corners.

\subsection{Modeling of the Brinkman number}

It must be remarked that the Brinkman number changes when the configuration changes from that of reference, as both the mean flow velocity and the peripheral heat flux change. Because of this, $\mathrm{Br}$ should be related to the objective functions. If $B r_{\text {ref }}$ is taken as that for sharp-cornered cross-sections, then

$$
B r=\left(\frac{\dot{\mathrm{m}}^{*}}{\mathrm{~A}_{\mathrm{c}}^{*}}\right)^{2} \frac{\mathrm{L}^{*}}{\dot{Q}^{*}} B r_{r e f}
$$

As done previously for the Poiseuille and Nusselt numbers, also the Brinkman number can be written as a function of the duct geometry, that is $B r=B r\left(R_{c}, \beta\right)$, yet the expression depends on the performance criterion investigated, as is demonstrated in the following for the case FG1a, where, by applying the restrictions on the normalized length and flow rate, one gets

$$
B r=\frac{1}{\mathrm{~A}_{\mathrm{c}}^{* 2} P_{h}^{* 2}} \frac{B r_{r e f}}{N u_{B r=0}^{*}} \frac{1+\sigma B r}{1+\sigma_{r e f} B r_{r e f}}
$$

Equation (13) can be solved for $\mathrm{Br}$ and yields

$$
\mathrm{B} r=\frac{B r_{r e f}}{\mathrm{~A}_{\mathrm{c}}^{* 2} P_{h}^{* 2} N u_{B r=0}^{*}\left(1+\sigma_{r e f} B r_{r e f}\right)-\sigma B r_{r e f}}
$$

It is to be noticed that the Brinkman number is thus a function of the aspect ratio $\beta$ and of the radius of curvature, but it also depends on the geometrical restriction, as both the nondimensional cross-sectional area and heated perimeter appear in Eq. (14).

Finally, a lower limit for the significance of viscous dissipation should be defined, both in terms of first and second law for all cases. To this purpose a difference of 5\% between the values of the relevant parameter (heat duty/ entropy generation due to heat transfer) with and without consideration of viscous dissipation was chosen as threshold. Although it is not shown here due to space constraints, the corresponding Brinkman numbers are one order of magnitude lower for entropy generation $\left(10^{-4}\right.$ instead of $10^{-3}$ for $\left.\beta=1\right)$. This is consistent with the underlying physics, as the parameter that accounts for entropy generation is more sensitive to the irreversibility linked to viscous dissipation.

\section{Results and discussion}

Several aspect ratios have been studied $(\beta=1.00,0.50,0.25,0.10$ and 0.03$)$, with reference Brinkman numbers that vary between $10^{-1}$ and $10^{-3}$, the upper limit of the interval investigated depending on the aspect ratio (the lower $\beta$, the lower $B r$ ) in order to have Nusselt numbers always higher than unity, so that convection is actually exploited. All calculations and plots are carried out through MATLAB ${ }^{\circledR}$.

\subsection{Case FG1a, $\beta=1, B_{\text {ref }}=0.1$}

As previously stated, results for the case FG1a only are presented here; this case refers to a fixed overall heat transfer area where the starting surface is replaced by others of the same basic geometry (Webb 1994), hence the need to place a constraint on either $a, P_{h}, D_{h}$ or $A_{c}$. The objective function to be maximized is the heat duty, which is expressed through an energy balance (Suzzi 2014)

$\dot{Q}=\dot{Q}_{B r=0}^{*} \frac{1+\sigma_{r e f} B r_{r e f}}{1+\sigma B r}$

while the constraints refer to the normalized values of length, temperature difference and flow rate which are kept fixed with respect to 
reference quantities, that is, $\dot{m}^{*}, L^{*}, \Delta T^{*}=1$. As previously stated, $B r$ changes its value with $R_{c}$ depending on the geometrical constraint and on the radius of curvature being investigated. The Brinkman numbers for $\beta=1$ and $B r_{r e f}=0.1$ are plotted in Fig. 2. It is plain to see how the geometric constraint is decisive in determining the actual Brinkman number associated with a given value of the radius of curvature. The plot shows that leaving the hydraulic diameter or the base size unchanged results in a steep increase of $B r$. Keeping $A_{c}$ fixed decreases $B r$, as this implies that the characteristic dimension $a$ must be higher than aref, and the constraint $\dot{m}^{*}=1$ results in a decrease of the mean velocity and of viscous dissipation with it. On the contrary, $D_{h}$ increases with $R_{c}$ if $a$ is fixed: then $D_{h}{ }^{*}=1$ means a diminution of the size of the channel $\left(a^{*}<1\right)$, which results in higher velocity gradients and, ultimately, viscous dissipation.

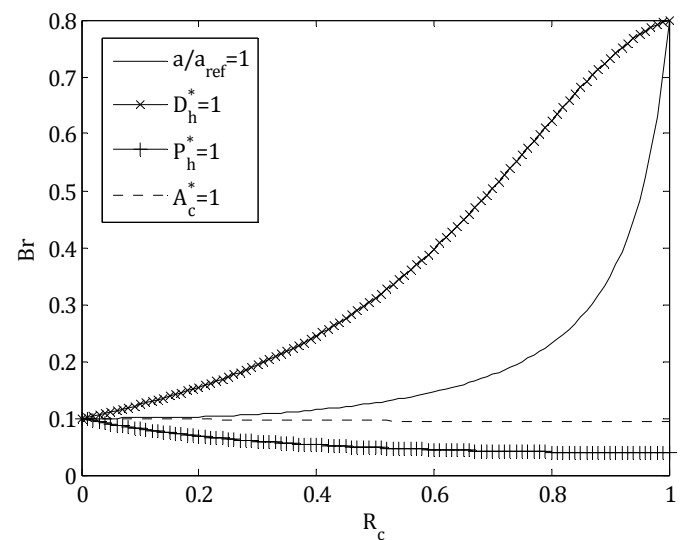

Figure 2 - Brinkman numbers for $\beta=1, \mathrm{Br}_{\mathrm{ref}}=0.1$, criterion FG1a.

This limits in practice the range of viable radii of curvature; in fact, for higher values of $B r_{r e f}$ the increase becomes asymptotical and yields negative values for higher $R_{c}$, which indicates that the flow would heat the channel due to frictional losses, instead of being heated.

The trends of the objective function $Q^{*}$ are plotted in Fig. 3 as a function of $R_{c}$.

Contrary to the case of $B r=0$ (no viscous dissipation), the $Q^{*}$ depends on the geometric constraint applied. Keeping the hydraulic diameter or the base side fixed results in strong increases in viscous dissipation with a steep drop in heat transfer rates (down to about 20\% of the reference case). On the other hand, the increase of $R_{c}$ gives an increase of up to $60 \%$ in the heat duty with respect to the reference configuration when the heated perimeter remains unchanged.

The case of fixed cross-sectional area exhibits a modest increase of $Q^{*}$ with the smoothing of the corners. The entropy generation number for $\beta=1$ is shown in Fig. 4.

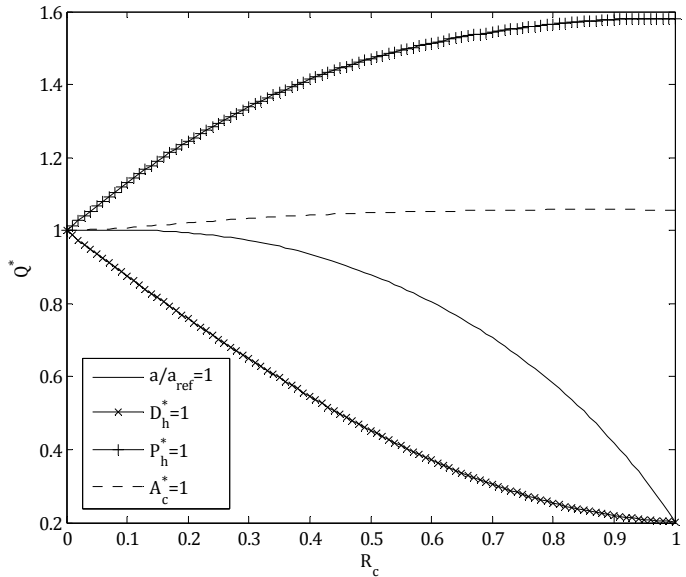

Figure 3 - Normalized heat duty for $\beta=1, \mathrm{Br}_{\mathrm{ref}}=0.1$.

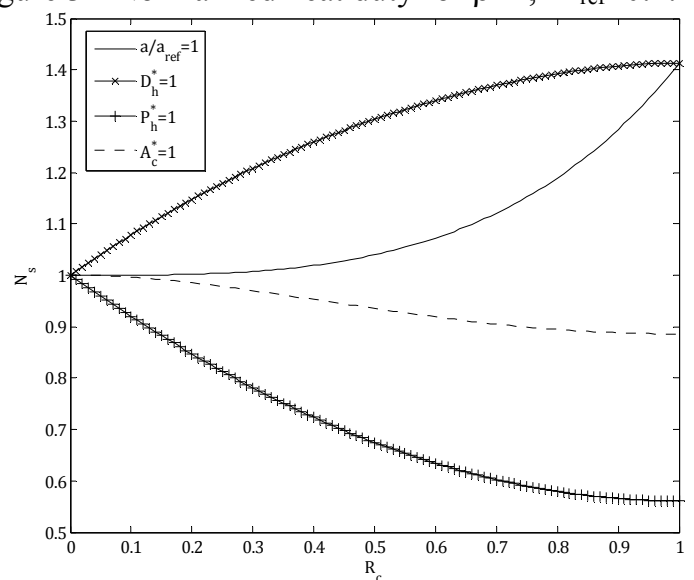

Figure 4 - Entropy generation number $\mathrm{N}_{\mathrm{S}}$ for $\beta=1$, $\mathrm{Br}_{\mathrm{ref}}=0.1$, case FGla.

Also in this case a fixed heated perimeter brings a decrease of $N_{s}$, of nearly $50 \%$ in the most favorable configuration $\left(R_{c}=1\right)$. This is in accordance with what was found for $Q^{*}$, and contrasts with the case of $B r=0$, when smoothing the corners always determines a decrease of the transferred power and in most cases a decrease of the entropy generation number for the same value of the reference irreversibility ratio (Suzzi, 2014). Smoothing the corners while keeping the cross-sectional area fixed also decreases entropy generation, albeit no more than $11 \%$, again for a unit radius of curvature. Applying the constraints on $a$ or $D_{h}{ }^{*}$, on the other hand, increases the 
generation of entropy in full accordance with the results for $Q^{*}$.

The two approaches, i.e. that using PECs and that using entropy generation numbers, give indications which may be combined together in order to have one single parameter that contains both pieces of information, as suggested by Zimparov (2001). One should be aware, however, that this is only one possible way: nothing prevents the designer from assigning a relative weight (determined by constraints pertaining to the problem being handled or stemming from the designer's experience) to each of them an adding them up to get another indicator.

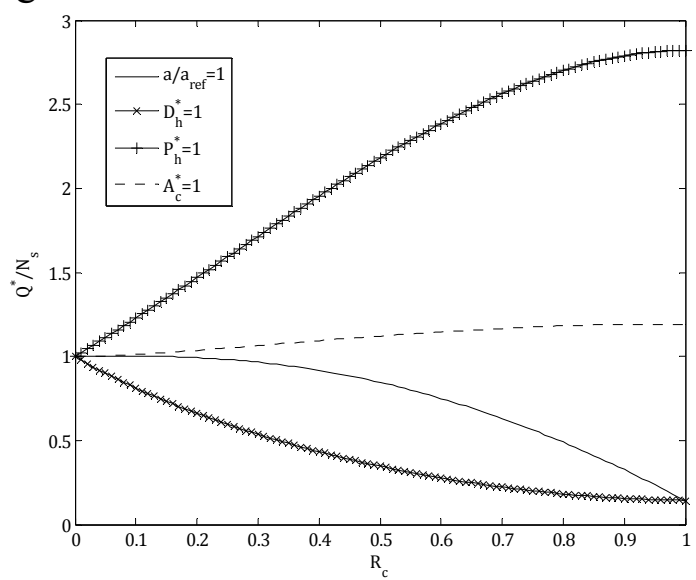

Figure 5 - Combined performance criterion for $\beta=1$, $\mathrm{Br}_{\mathrm{ref}}=0.1$, case FG1a.

In the approach by Zimparov, a ratio of the heat duty to $\mathrm{N}_{\mathrm{S}}$ is calculated, with values larger than one corresponding to more favorable configurations than the reference for both criteria. The result is shown in Fig. 5 .

The increase of the radius of curvature is clearly of great benefit when the geometrical constraint is on the heated perimeter, is more modest for the case of a fixed cross-sectional area and is definitely unfavorable for the remaining two constraints.

If the $\phi_{\text {ref }}$ is varied between $10^{-3}$ and $10^{3}$, the trends of the objective functions and their combination are very similar, contrary to what happens when viscous dissipation is negligible.

To understand why, Eq. (11) is to be considered; it is clear from it that $\mathrm{N}_{S}$ results from a weighted average of $N_{T}$ and $N_{P}$, and their dependence on the radius of curvature of the corners is shown in Fig. 6 (a) and 6(b).
The trends of the two quantities are almost the same both qualitatively and quantitatively, and they do not depend on $\phi_{r e f}$. As a consequence, since $N_{S}$ is obtained from their weighted average, it cannot vary much, regardless of the magnitude of the weight of each term.

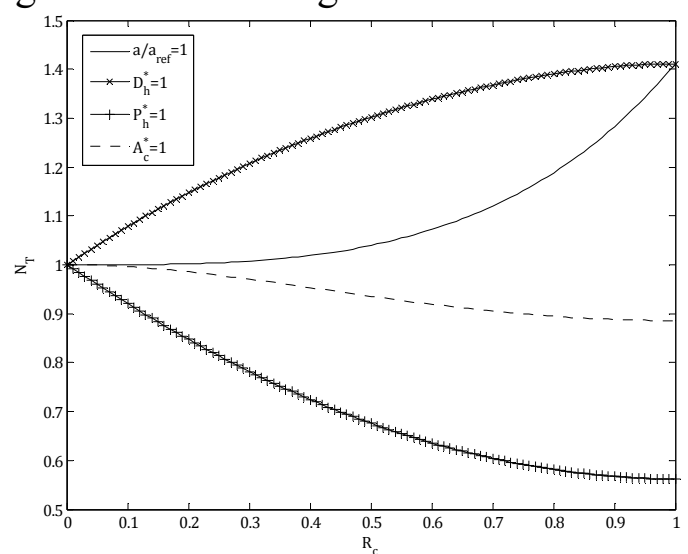

(a)

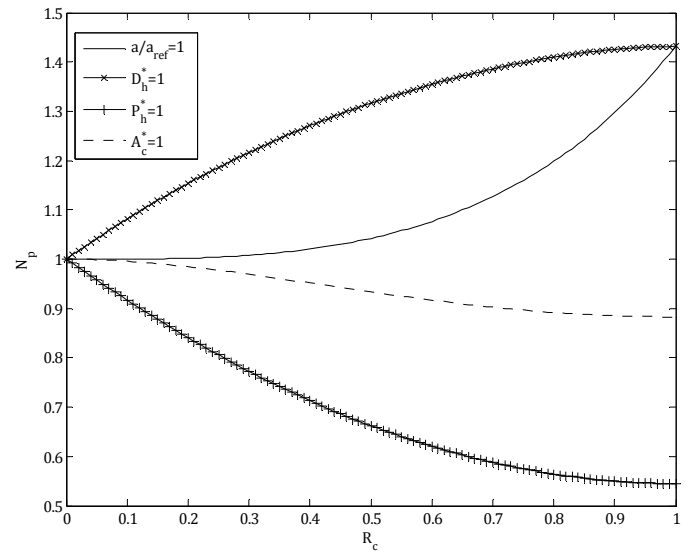

(b)

Figure 6 - Trends of $N_{T}$ and $N_{P}$ as a function of $R_{c}$, for $\beta=1, \mathrm{Br}_{\text {ref }}=0.1$, case FG1a.

The above results suggest that viscous dissipation is dominant over any other effect on the performance of the microchannel for these $B r_{r e f}$ and any measure mitigating its effects may strongly benefit the heat transfer.

\subsection{Case $\beta=1, B r_{\text {ref }}=0.01, B r_{\text {ref }}=0.001$}

When the values of the $B r_{\text {ref }}$ decrease, a waning of viscous dissipation effects and a resemblance of the trends of $Q^{*}$ and $\mathrm{N}_{\mathrm{s}}$ to those for $B r=0$ are to be expected.

Actually, a change from the trend of Fig. 3 is seen for $Q^{*}$ at $B r_{r e f}=0.01$, as shown in Fig. 7 .

Only for constrained heated perimeter smoothing the corners is beneficial, but there is a maximum for $R_{c}=0.39$ and even so the increase is a modest $3 \%$. 
All other restrictions result in lower values of $Q^{*}$ for ducts with smoothed corners, with decreases down to $12 \%$ for constrained $D_{h}{ }^{*}$ and $a$. On the contrary, only a shortening of the range of variability is experienced for Ns, which is not shown. Again, this testifies that irreversibilities have a far larger impact on entropy generation than on $Q^{*}$.

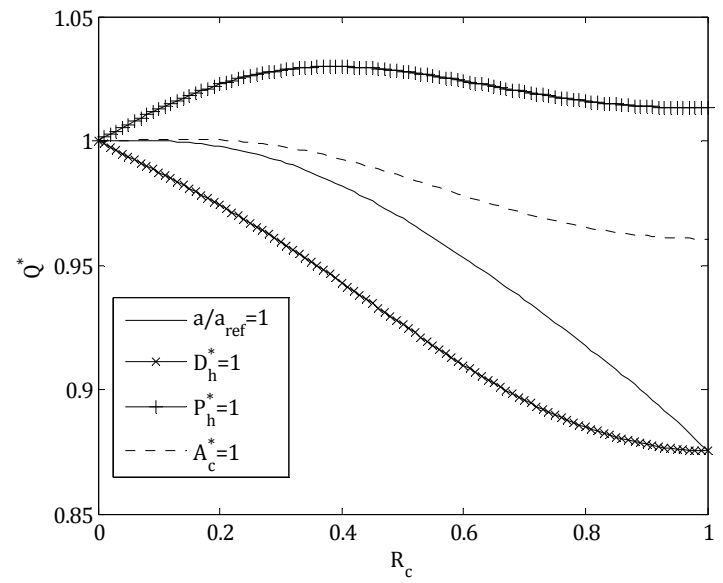

Figure 7 - Normalized heat duty for $\beta=1, \mathrm{Br}_{\mathrm{ref}}=0.01$, case FGla.

Since the range of variability of $Q^{*}$ is far more restricted than for $B r_{r e f}=0.1$, the combination parameter $Q^{*} / N_{S}$ resembles both in shape of the curves and in ranges of variability the trends of $N_{S}$, see Fig. 8.

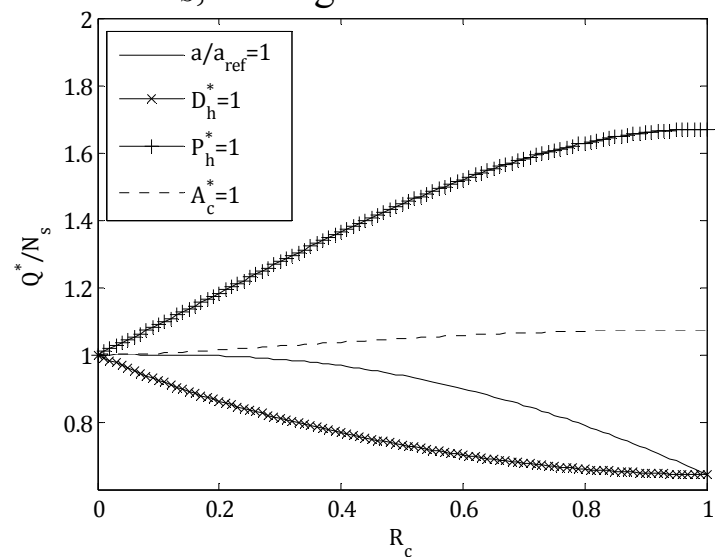

Figure 8 - Combined performance criterion for $\beta=1$, $\mathrm{Br}_{\text {ref }}=0.01$, case FG1a.

The maximum value of the combined parameter is again achieved for fixed heated perimeter and unit radius of curvature, but is much lower than before; since the incidence of viscous dissipation (as exemplified by $B r$ ) decreases, and so does the maximum benefit that can be gained by smoothing the channel's corners. In this case, the influence of $\phi_{\text {ref }}$ is no longer negligible: the highest value of $\phi_{\text {ref }}$ ensure about $25 \%$ more increase for $R_{c}=1$ as compared with the lowest $\phi_{\text {ref }}$.

The Brinkman number has a much narrower range of variability than for $B r_{r e}=0.1$ : although it is not shown here, the maximum and minimum values are achieved for $R_{c}=1$ and are 0.02 and 0.006 respectively.

The trends above are confirmed for $\mathrm{Br}=0.001$, and the curves for $Q^{*}$. are more similar to those obtained for $B r=0$.

By the same token, the trends of $B r\left(R_{c}\right)$ flatten as $B r_{\text {ref }}$ decreases, showing the loss of dependence of viscous dissipation on $R_{c}$ and on the geometrical constraint.

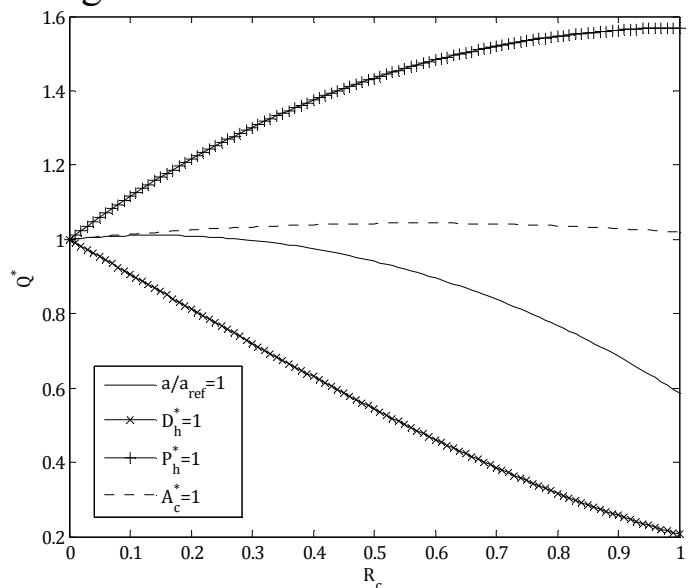

Figure 9 - Normalized heat duty for $\beta=0.5, \mathrm{Br}_{\text {ref }}=0.1$.

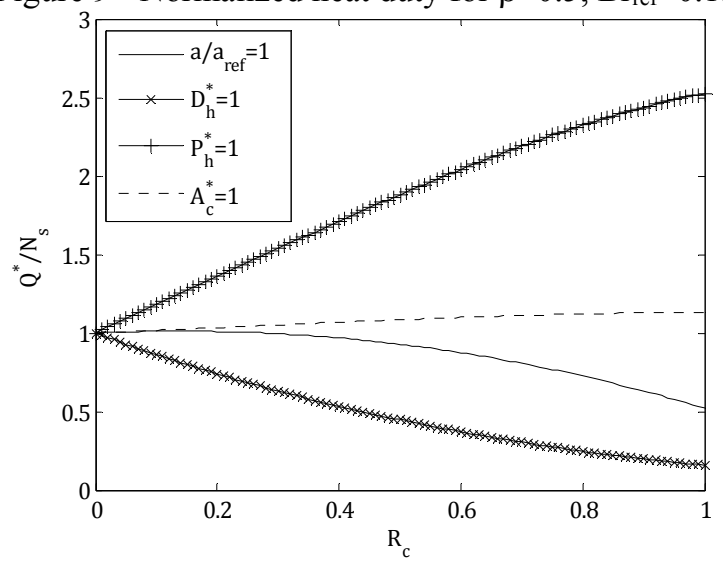

Figure 10 - Combined performance criterion for $\beta=0.5$, $\mathrm{Br}_{\mathrm{ref}}=0.1$.

\subsection{Case FG1a, $\beta<1, B_{\text {ref }}=0.1$}

If $\beta$ decreases, the influence of rounding the corners still has the same effects as for $\beta=1$, although the range of variability of the parameters decreases, as shown in Figs. 9 and 10 for $Q^{*}$. and $Q^{*} / N_{S}$ for $\beta=0.5$. It can be noticed that, although the heat duty for fixed $P_{h}{ }^{*}$ is almost the same as for $\beta=1$, the combined parameter exhibits a decrease for the 
same constraint, indicating that the entropy generation is still favorably affected by smoothing the corners, but to a lesser extent.

\section{Conclusions}

The main conclusions of this work can be summarized as follows:

- for a given $B r_{r e f}$ the corresponding Brinkman number for the objective function changes depending on the PEC and constraints considered; constraints on $D_{h}{ }^{*}$ and $a / a_{\text {ref }}$ cause the largest increases;

- as a consequence $Q^{*}$ depends on the geometrical constraint, unlike the case with no viscous dissipation;

- the trend of $N_{S}$ is concordant with that of $Q^{*}$, contrary to when $B r_{\text {ref }}=0$;

- the combined parameter shows that the best results are obtained for $P_{h}{ }^{*}=1$ and maximum smoothing for all $\beta$; also for $A_{c}{ }^{*}=1$ smoothing is beneficial, albeit to a lesser extent;

- the results are unaffected by $\phi_{r e f}$ when $\beta=1$; for lower aspect ratios there is a variation in the results of about $25 \%$ for $10^{-3} \leq \phi_{\text {ref }} \leq 10^{3}$.

- as Brref decreases, Q* shows a behavior closer to that for vanishing viscous dissipation, while $N_{S}$ continues to be affected, although the ranges of variability decrease;

- if $\beta<1$ and $B r_{\text {ref }}=0.1, Q^{*}$ changes little from the case $\beta=1$, whereas $Q^{*} / N_{S}$ has the same trend but lower values, showing that $N_{S}$ is more affected by the change in $\beta$.

- The results can be applied to the design of heat sinks for electronics, gas coolers and regenerators for micro gas turbines.

\section{References}

Bejan A., 1980. Second law analysis in heat transfer, Energy, 5 721-732.

Bejan A., 1984. Entropy Generation Through Heat and Fluid Flow Wiley, New York 1982.

Chakraborty S., Ray S. 2011. Perf. Opt. of lam. fully-dev. flow through square ducts with rounded corners, Int. J. Th. Sci. 50, 2522-2535
Dessiatoun S., Cetegen E., Ohadi M., Choo K., 2012. Next Gen. $\mu$ ch. $H X$ Springer, Berlin.

Han Y., Liu Y., Li M., Huang j., 2012. A review of devel. of $\mu$-channel heat exch. App. in air-cond. sys., En. Proc., 14, 148-153.

Jaluria J. 2007. Design and Opt. of Thermal Systems 2nd Edition CRC Press, Boca Raton

Kew P.A., Reay D.A., 2011. Compact/microheat exch. - Their role in heat pumping equip. App. Th. Eng. 31, 594-601.

Lorenzini M. 2013. The influence of visc. diss. on thermal perf. of $\mu$ channels with rounded corners. Houille Blanche 4 64-71

Lorenzini M., Morini G.L. 2011. Single phase lam. forced conv. in $\mu$ channels with rounded corners. Heat Tr. Eng. 32 (13-14) 1108-1116

Morini G.L., 2005. The Viscous Heating in Liquid Flows in Microchannels, Int. J. Heat and Mass Transfer, 48, 3637-3647.

Petkov V. M., Zimparov V.D., Bergles A. E. 2014 Perf. Eval. of ducts with non-circ.shapes: lam. fully dev. fl. Int. J. Th. Scien. 79 220-228.

Ray S., Misra D. 2010. Lam. Ful. dev. fl. thr. sq. and Eq. Triang. ducts w. round. corn. subj. to $\mathrm{H} 1$ and $\mathrm{H} 2$ b.c. Int. J. Th. Sc. 49 1763-1775.

Suzzi N. 2014. Evaluation of thermalhydraulic characteristics of microchannels with rounded corners, MS Thesis (in Italian).

Webb R.L. and Bergles A.E., 1983. Perf. Eval. Crit. for selection of heat transfer surface geom. used in: Low Reynolds No. flow HX, Hemisph. Publ. Corp. Washington, 735-742.

Webb R.L., 1981. Perf. Eval. Crit. for use on enh. heat tr. Surf. in HX. design Int. J. Heat Mass Tr. 24,715-726.

Webb R.L., 1994. Principles of Enhanced Heat Transfer Wiley, New York.

Zimparov V.D. 2001 Ext. PEC for enhanc. heat tr. surfaces: heat transf. thr. duc. w. con. h. fl. IJHMT 44 169-180. 